\title{
Genomic Analysis Reveals Novel Specific Metastatic Mutations in Chinese Clear Cell Renal Cell Carcinoma
}

\author{
Hui Meng $\mathbb{D},{ }^{1}$ Xuewen Jiang, ${ }^{1}$ Jianfeng Cui $\mathbb{D}^{1},{ }^{\text {Gang }}$ Yin, ${ }^{1}$ Benkang Shi, ${ }^{1}$ Qi Liu $\mathbb{D},{ }^{2}$ \\ He Xuan, ${ }^{2}$ and Yu Wang iD ${ }^{3}$ \\ ${ }^{1}$ Department of Urology, Qilu Hospital of Shandong University, 107 Jinan Culture Road, Jinan, 250012 Shandong, China \\ ${ }^{2}$ Life Healthcare Medical Laboratory Co., Ltd., Hangzhou, 310052 Zhejiang, China \\ ${ }^{3}$ Reproductive Medicine Center, Department of Obstetrics and Gynecology, Qilu Hospital of Shandong University, 107 Jinan \\ Culture Road, Jinan, 250012 Shandong, China
}

Correspondence should be addressed to Yu Wang; yuerjade@163.com

Received 22 April 2020; Revised 3 July 2020; Accepted 17 August 2020; Published 30 September 2020

Academic Editor: Bhaskar Dasgupta

Copyright (c) 2020 Hui Meng et al. This is an open access article distributed under the Creative Commons Attribution License, which permits unrestricted use, distribution, and reproduction in any medium, provided the original work is properly cited.

Clear cell renal cell carcinoma (ccRCC) accounts for more than $75 \%$ of renal cell carcinoma. Nearly $25 \%$ of ccRCC patients were diagnosed with metastasis. Though the genomic profile of ccRCC has been widely studied, the difference between localized and metastatic ccRCC was not clarified. Primary tumor samples and matched whole blood were collected from 106 sporadic patients diagnosed with renal clear cell carcinoma at Qilu Hospital of Shandong University from January 2017 to November 2019, and 17 of them were diagnosed with metastasis. A hybridization capture-based next-generation sequencing of 618 cancer-related genes was performed to investigate the somatic and germline variants, tumor mutation burden (TMB), and microsatellite instability (MSI). Five genes with significantly different prevalence were identified in the metastatic group, especially TOP1 (17.65\% vs. $0 \%)$ and SNCAIP (17.65\% vs. $0 \%)$. The altered frequency of PBRM1 (0\% vs. $27 \%)$ and BAP1 (24\% vs. $10 \%)$ differed between the metastatic and nonmetastatic groups, which may relate to the prognosis. Of these 106 patients, 42 patients (39.62\%) had at least one alteration in DNA damage repair (DDR) genes, including $58.82 \%$ of metastatic ccRCC patients and $35.96 \%$ of ccRCC patients without metastasis. Ten pathogenic or likely pathogenic (P/LP) variants were identified in 11 sporadic clear cell renal cell carcinoma patients $(10.38 \%)$, including rarely reported $A T M(\mathrm{n}=1), M U T Y H(\mathrm{n}=1), N B N(\mathrm{n}=1), R A D 51 D(\mathrm{n}=1)$, and BRCA2 ( $\mathrm{n}=1)$. No significant difference in the ratio of P/LP variant carriers or TMB was identified between the metastatic and nonmetastatic groups. We found a unique genomic feature of Chinese metastatic ccRCC patients with a higher prevalence of alterations in DDR, TOP1, and SNCAIP. Further investigated studies and drug development are needed in the future.

\section{Introduction}

Renal cell carcinoma (RCC) is the seventh most common cancer in men and the ninth most common cancer in women, accounting for 2 to 3 percent of all adult malignancies. The estimated incidence and mortality of RCC in China in 2015 were 66,800 and 23,400, respectively [1]. RCC comprises more than 10 histological and molecular subtypes, with differences in the histological pattern, clinical course, and genomic feature. Of these types, clear cell renal cell carcinoma (ccRCC) accounts for the most proportion (75\%-80\%) and has a worse prognosis [2]. To date, surgery, especially radical nephrectomy, is still the most effective treat- ment for localized ccRCC patients. However, nearly $25 \%$ of RCC patients were diagnosed with metastasis and unable to take surgery to remove the tumor [3]. Besides, RCC is commonly resistant to chemotherapy and radiotherapy, so the 5 -year survival was poor. A clear understanding of the genomic features of metastatic ccRCC will help us to select personalized treatments and develop new effective drugs.

With the widespread next-generation sequencing technology, the genomic landscape of ccRCC has been revealed by many studies [4]. ccRCC was mainly characterized by the loss of chromosome $3 \mathrm{p}$, and alterations of genes involved in this location, especially $V H L, S E T D 2, P B R M 1$, and BAP1, are suggested as the driver events of ccRCC [5]. Research 
on the genomic feature of Chinese ccRCC patients is deficient, and limited results showed a similar driver role of $V H L, S E T D 2, P B R M 1$, and BAP1 genes and unique genes involved in the ubiquitin-mediated proteolysis pathway [6]. A comparison of genetic differences between localized and metastatic ccRCC has not been well depicted yet. A significant higher mutated frequency of TP53 was found in the metastatic ccRCC, but no difference in other genes or tumor mutation burden was found [7].

We conducted a genomic study to determine the difference in the gene prevalence between Chinese nonmetastatic and metastatic ccRCC patients and discovered potential related to metastasis.

\section{Materials and Methods}

2.1. Biospecimen Collection and Clinical Data. Primary tumor samples and matched whole blood were collected from 106 sporadic patients diagnosed with renal clear cell carcinoma at Qilu Hospital of Shandong University from January 2017 to November 2019. 17 of them were diagnosed with metastasis, and the rest was localized ccRCC. Written informed consent was obtained from each patient for collection and further publication of their clinical details and tumor genomic profiles. All enrolled patients did not receive any neoadjuvant treatment before sample collection, including chemotherapy, anti-VEGFR or mTOR therapy, and immunotherapy. All tumor samples were pathologically reviewed to have at least $60 \%$ tumor cells. Clinical data were collected, including patient ID, gender, age, and metastatic status at diagnosis (Supplementary Table S1).

2.2. Target Next-Generation Sequencing. For the matched blood and tumor samples, germline DNA (gDNA) was isolated using the DNeasy Blood \& Tissue Kit (Qiagen, 69504) according to the manufacturer's instruction. $100 \mathrm{ng}$ of gDNA was sheared with a Covaris E210 system (Covaris, Inc.) to target fragment sizes of $200 \mathrm{bp}$. We performed library preparation for tumor gDNA and matched germline gDNA using the Accel-NGS 2S DNA Library Kit (Swift Biosciences, Inc.) and target enrichment using the xGen Lockdown Probe kit (IDT, Inc.). The custom xGen Lockdown Probe was synthesized by IDT, Inc. for the exons and parts of introns of 618 genes of interest (Supplementary Table S2). Samples underwent paired-end sequencing on an Illumina Nextseq CN500 platform (Illumina Inc.) with a $150 \mathrm{bp}$ read length and mean coverage of $1000 \mathrm{x}$ at a $95 \%$ capture rate and $40 \%$ dup rate.

2.3. Data Analysis. Raw sequencing data were aligned to the reference human genome (UCSC hg19) through BurrowsWheeler Aligner and producing a binary alignment/map (BAM) file. After the duplicate removal and local realignment, the Genome Analysis Toolkit (GATK) was used for single-nucleotide variation (SNV) and short insertion/deletion (indel) calling. Variants were annotated using the ANNOVAR software tool. Variants identified in gDNA from buffy coat fraction aliquots with allele fraction (AF) beyond $25 \%$ were determined as germline variants. The interpreta-
TABLE 1: Clinical characteristics of 106 ccRCC patients.

\begin{tabular}{lcc}
\hline Characteristics & $\begin{array}{c}\text { Metastatic group } \\
(n=17)\end{array}$ & $\begin{array}{c}\text { Nonmetastatic group } \\
(n=89)\end{array}$ \\
\hline Age & $57(34-74)$ & $55(25-86)$ \\
$\quad$ Median & & \\
Sex & 14 & 64 \\
$\quad$ Male & 3 & 25 \\
Female & & \\
Stage & 0 & 38 \\
I & 1 & 13 \\
II & 7 & 27 \\
III & 9 & 11 \\
IV & & \\
\hline
\end{tabular}

tion of germline variants followed the standards and guidelines of the American College of Medical Genetics and Genomics and the Association for Molecular Pathology (ACMG/AMP) and was independently reviewed by two genetic consultants [8]. Somatic variants with AF beyond $1 \%$ were generated from each tumor gDNA by removing the germline variants and further annotated according to the Catalog of Somatic Mutations in Cancer (COSMIC) database. The functional classification of each somatic mutation followed the interpretation and reporting standards and guidelines recommended by the Association for Molecular Pathology, American Society of Clinical Oncology, and College of American Pathologists (ASCO/CAP) [9]. The tumor mutation burden of each sample was calculated according to a published and widely applied method [10].

2.4. Statistical Analysis. Differential mutation analysis was performed using the Chi-squared test or Fisher exact test under a dominant model. Two-sided $P$ values less than 0.05 were considered to be statistically significant. All analyses were performed using SPSS 25.0 software.

\section{Results}

3.1. Somatic Mutation Landscape of ccRCC with/without Metastasis. The clinical data of 106 enrolled ccRCC patients of primary kidney tissues (comprising of 89 localized and 17 metastatic ccRCC) is shown in Table 1.

The prevalence of somatic altered genes in metastatic and nonmetastatic ccRCC patients is shown in Figure 1. We found that the most frequently altered genes in metastatic ccRCC were VHL (47\%), BAP1 (24\%), TOP1 (18\%), and SNCAIP (18\%) in metastatic patients (Figure 1(a)). The most altered genes in nonmetastatic ccRCC were $V H L$ (57\%), PBRM1 (27\%), SETD2 (11\%), and TP53 (11\%), respectively (Figure 1(b)). Meanwhile, the median TMB of the metastatic and nonmetastatic ccRCC patients was 7.56 and 6.67 mutations/Mb, respectively, with no significant difference (Figure 1(c)). No microsatellite instability-high (MSI-H) tumor was identified in the 106 ccRCC samples.

We used the Circos plot to display the gene mutations on the chromosome and found that the somatic mutations were 


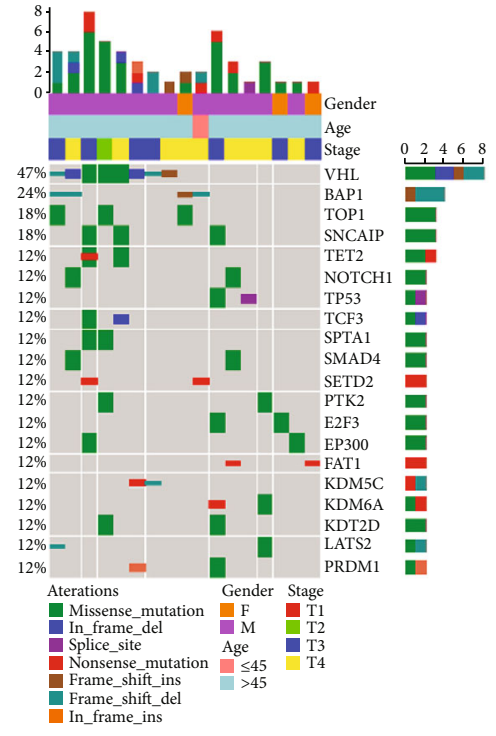

(a)

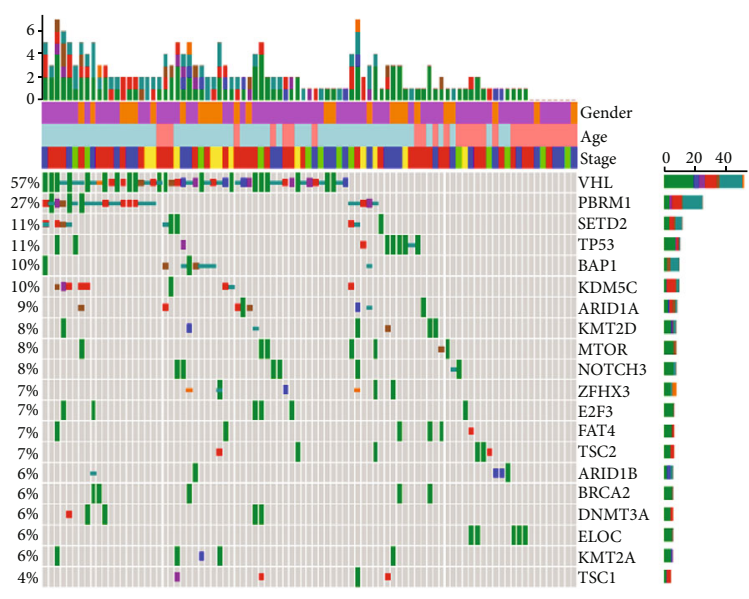

(b)

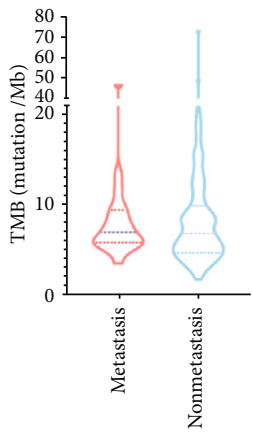

(c)

FIgURE 1: The genomic landscape of somatic mutations in ccRCC with/without metastasis. (a) Oncoprint illustrations of somatic alterations in metastatic ccRCC by gene frequency. (b) Oncoprint illustrations of somatic alterations in nonmetastatic ccRCC by gene frequency. (c) The difference in tumor mutation burden (TMB) between the metastasis and nonmetastasis groups.

widely distributed in the nonmetastatic ccRCC but relatively concentrated in the metastatic ccRCC in contrast. The four mutational patterns with the highest frequency in ccRCC patients were missense (metastatic versus nonmetastatic group, $68.99 \%$ vs. $54.91 \%$ ), complex substitution (16.28\% vs. $19.85 \%)$, synonymous ( $9.30 \%$ vs. $15.03 \%$ ), and splice site (2.33\% vs. $5.20 \%$ ) (Figures $2(\mathrm{a})$ and $2(\mathrm{~b})$ ). The prevalence of altered genes of the total of 106 ccRCC patients was compared with The Cancer Genome Atlas (TCGA) data. Four genes with both high mutation frequency and low $P$ value were identified, including ZFHX3 (our cohort versus TCGA, $6.60 \%$ vs. $1.17 \%$ ), NOTH3 (6.60\% vs. $1.41 \%$ ), ARID $1 B$ (5.66\% vs. $0.94 \%$ ), and TSC2 (5.66\% vs. $0.94 \%$ ) (Figure 2(c)). Notably, a significantly higher frequency of altered E2F3 was identified in our cohort (7.55\% vs. $0 \%$ ) (Supplementary Table S3).

3.2. Differences in the Prevalence of Altered Genes between ccRCC with or without Metastasis. Next, we investigated the different frequencies of altered genes between the metastatic and nonmetastatic groups (Figure 3(a)). The metastatic and nonmetastatic groups harbored 191 and 40 genes with different mutated frequencies, respectively. The top ten unique mutated genes involved in the nonmetastatic cohort (Figure 3(b)) were PBRM1, NOTCH3, MTOR, TSC2, FAT4, KMT2A, ELOC, TSC1, SPEN, and PTPRT. On the contrary, a different prevalence of TOP1, SNCAIP, PRDM1, CDKN1A, WRN, TGFBR1, TEK, TCF7L2, SPOP, and SOX9 was involved in the metastatic cohort genes (Figure 3(b) and Supplementary Table S4). Reactome pathway analysis of the genes with different prevalence showed that the nonmetastatic group was characterized by gene sets associated with developmental biology, signal transduction signaling, PI3K/AKT activation, and cancer classic signaling pathways (Figure 3(c)), while the metastatic group was characterized by gene sets associated with homology recombination repair, DNA repair, and DNA double-strand break signaling pathway (Figure 3(c)).

3.3. Details on Genes with Different Prevalence in ccRCC with or without Metastasis. Specifically, we identified 101 genes with different prevalence between the metastatic and nonmetastatic cohorts. Lollipop plots showed the distribution of $V H L, P B R M 1$, and BAP1 mutations, and truncating was the most common mutation types in three genes, followed by missense and inframe indels (Figure 4(a)). Notably, TOP1 and SNCAIP variants were only identified in the metastatic group $(P=0.0035$, Table 2$)$. The distribution of TOP1 and SNCAIP variants is illustrated in Figure 4(b). On the contrary, $P B R M 1$ was only mutated in the nonmetastatic group (Figure 4(c)). No significant difference was found in VHL and BAP1 between the two groups.

3.4. Somatic Alterations in DNA Damage Repair (DDR) Pathway. Of these 106 patients, 42 patients (39.62\%) had at least one alteration in DDR genes, including $58.82 \%$ of metastatic ccRCC patients (10/17) and 35.96\% of ccRCC patients without metastasis (32/89) (Figure 5(a)). The most common mutational pathway was the homology recombination (HR) repair pathway $(22.22 \%)$, followed by the mismatch repair (MMR) pathway (8.64\%, Figure 5(b)).

Among the DDR gene alterations, $22.64 \%$ of them were pathogenic. Specifically, the DDR genes with the most known or likely deleterious variants were TP53 and PTEN (Figure 5(c)).

3.5. Germline Mutations in ccRCC. Ten pathogenic or likely pathogenic $(\mathrm{P} / \mathrm{LP})$ variants were identified in 11 sporadic clear cell renal cell carcinoma patients $(10.38 \%)$. None of 



(a)

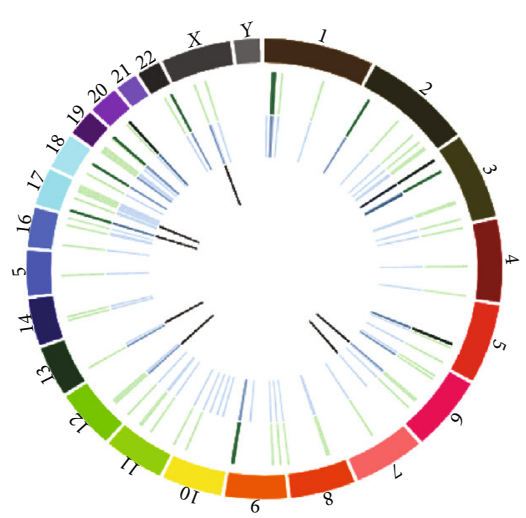

(b)

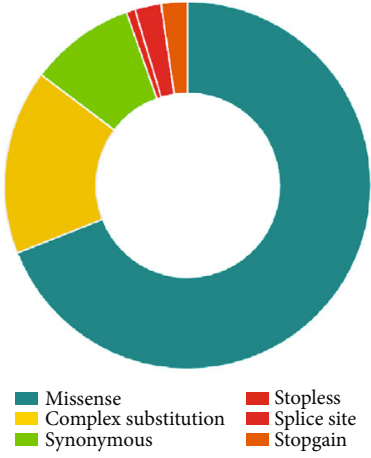

Synonymous

Stopgain

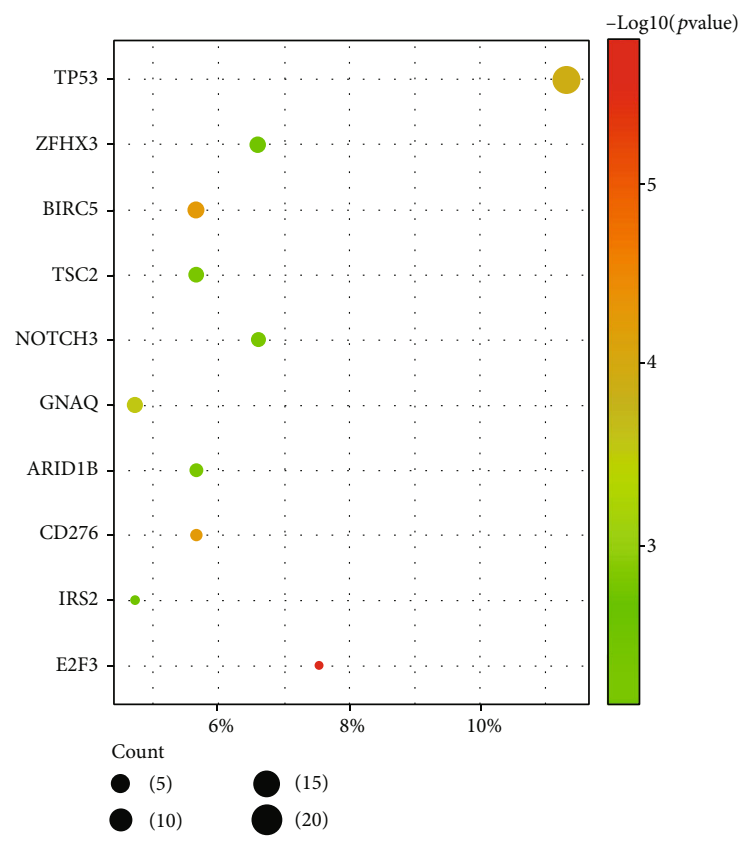

(c)

FIGURE 2: Comparison of somatic mutation patterns in ccRCC with/without metastasis on the chromosome and the frequency of differential mutation genes in the TCGA ccRCC cohort. The Circos plot and pie plot display gene mutational patterns on the chromosome and frequency of every variant (a) in the nonmetastatic group and (b) in the metastatic group. (c) The diagram displays mutational gene frequencies of the total 106 ccRCC patients (Fisher's exact test), compared with the TCGA data.

the P/LP carriers was with bilateral or multiple renal masses (Table 3). Three patients were with a family cancer history, but none had familial renal cell cancer. The mean age and median age at diagnosis of identified P/LP carriers were 50.72 and 50 years, respectively. Only 4 P/LP carriers (36.36\%) were diagnosed below 46 years old. There were no significant differences in the age at diagnosis between P/LP carriers and noncarriers. Except $V H L$ and $F H$ genes, which have been widely identified in hereditary kidney cancer, we found P/LP variants in rarely reported ATM $(n=2), M U T Y H$ $(n=2), N B N(n=2), R A D 51 D(n=1)$, and BRCA2 $(n=1)$ genes in our cohort. No significant difference in the P/LP variant carriers' ratio was identified between the metastatic and nonmetastatic groups.

${ }^{*}$ Patients 022, 024, and 092 were ccRCC with metastasis. *Two sporadic patients had the same ATM variants.

\section{Discussion}

The genomic differences between Chinese metastatic and nonmetastatic ccRCC have not been revealed. Compared with TCGA, a higher prevalence of ZFHX3, NOTH3, $A R I D 1 B, T S C 2$, and E2F3 was identified in our cohort, suggesting a difference in the genomic feature between Chinese and Caucasian ccRCC patients. A past research found a prognostic value of E2f3 expression in ccRCC, which was significantly associated with tumor size, metastasis, lymph node metastasis, and tumor stage [11].

The genomic profile of ccRCC has been widely studied and is mainly characterized by the loss of chromosome $3 p$ [5]. Alterations of genes involved in this location, especially VHL, SETD2, PBRM1, and BAP1, are suggested as the driver events of ccRCC (Supplementary Table S5). Interestingly, we 


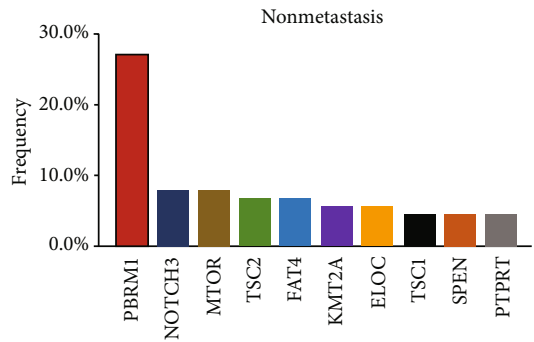

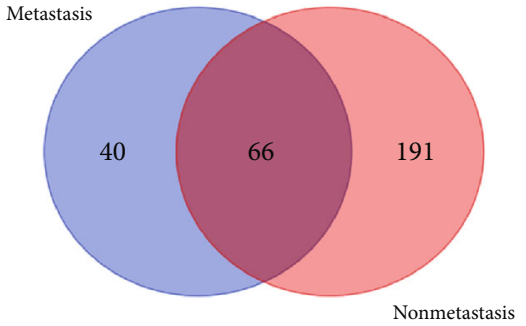

(a)

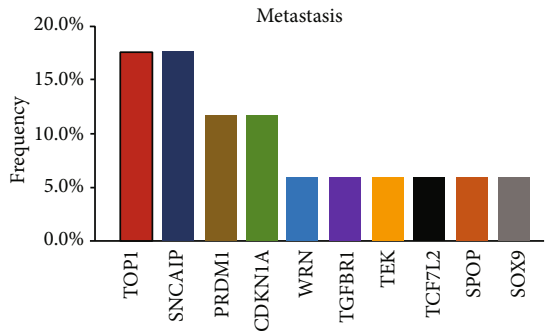

(b)

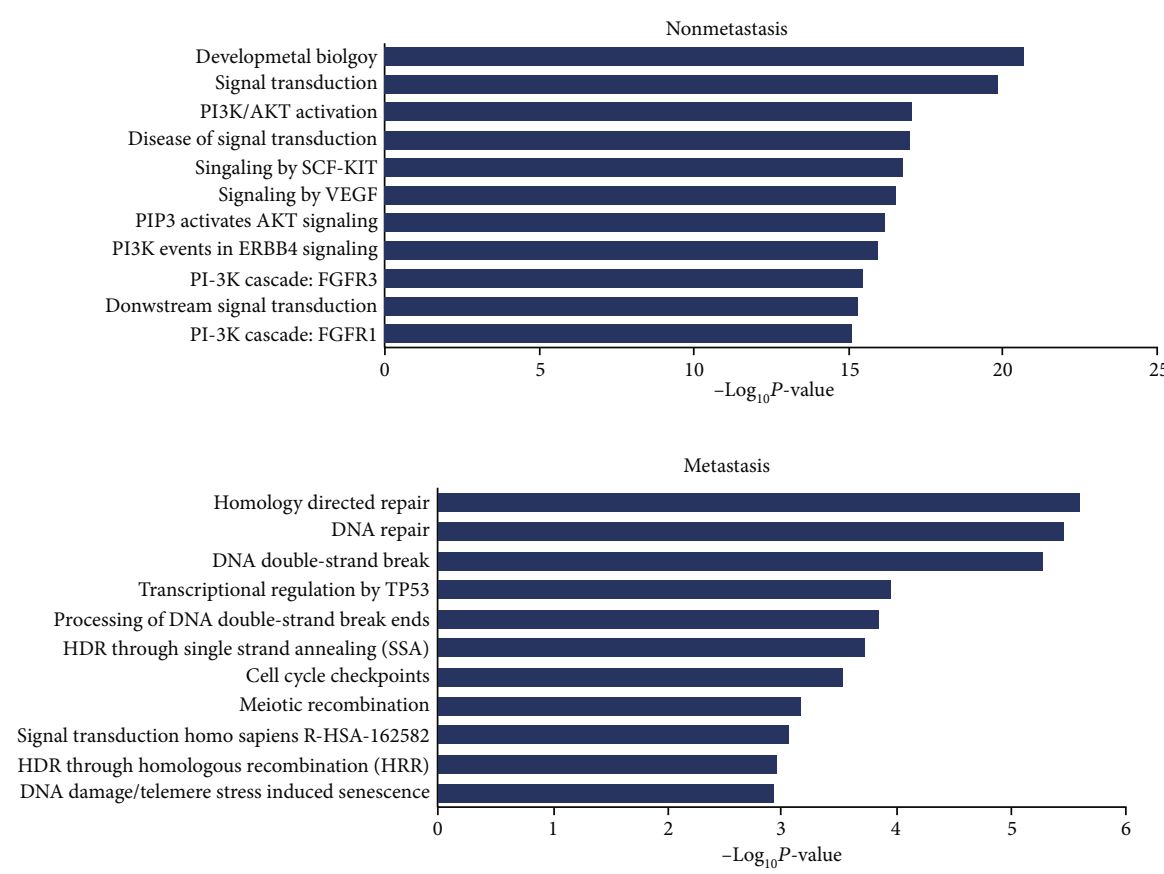

(c)

Figure 3: Analysis of genes with different prevalence in the nonmetastatic and metastatic groups. (a) Venn diagram illustrated the overlap and independently mutated genes between the nonmetastatic and metastatic groups. (b) The frequency of genes with different prevalence in the nonmetastatic (bottom) and metastatic (top) groups. (c) Reactome pathway analysis of mutated genes with different prevalence in the nonmetastatic (bottom) and metastatic (top) groups.

only identified $P B R M 1$ variants in the nonmetastatic ccRCC group (27\%); on the contrary, a higher $B A P 1$ prevalence in the metastatic ccRCC group (24\% vs. 10\%). BAP1 mutation is associated with worse survival in ccRCC, and PBRM1mutated patients had a favorable survival instead [12]. Differences in the prevalence of these two genes between localized and metastatic ccRCC may contribute to the different prognosis effects on survival. Meanwhile, PBRM1 alterations may correlate with the sensitivity of immune checkpoint therapy in ccRCC, though there are controversial results on its immunogenic effects $[13,14]$.
We only identified TOP1 and SNCAIP alterations in the metastatic ccRCC, which was not reported before. TOP1 encodes topoisomerase I (Top1), which is involved in the process of DNA replication and chromosomal recombination. As depicted by the immunochemistry analysis, Top1 expression was elevated in $23.5 \%$ of RCC, and increased expression was associated with a higher grade (grades 3 and 4) [15]. Only $0.7 \%$ of ccRCC patients had TOP1 alterations in the TCGA database, and all alterations were missense both in TCGA and our cohort. Camptothecin and related drugs, including irinotecan, were designed to inhibit tumor cell 


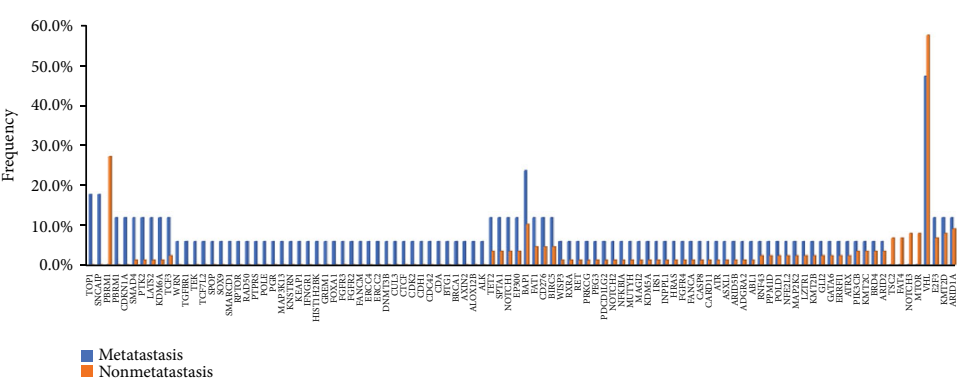

(a)

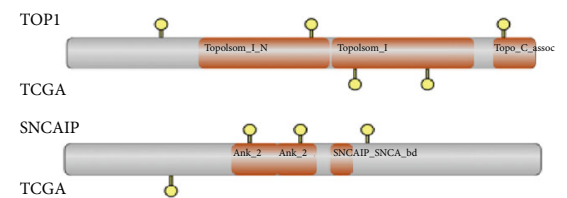

(b)
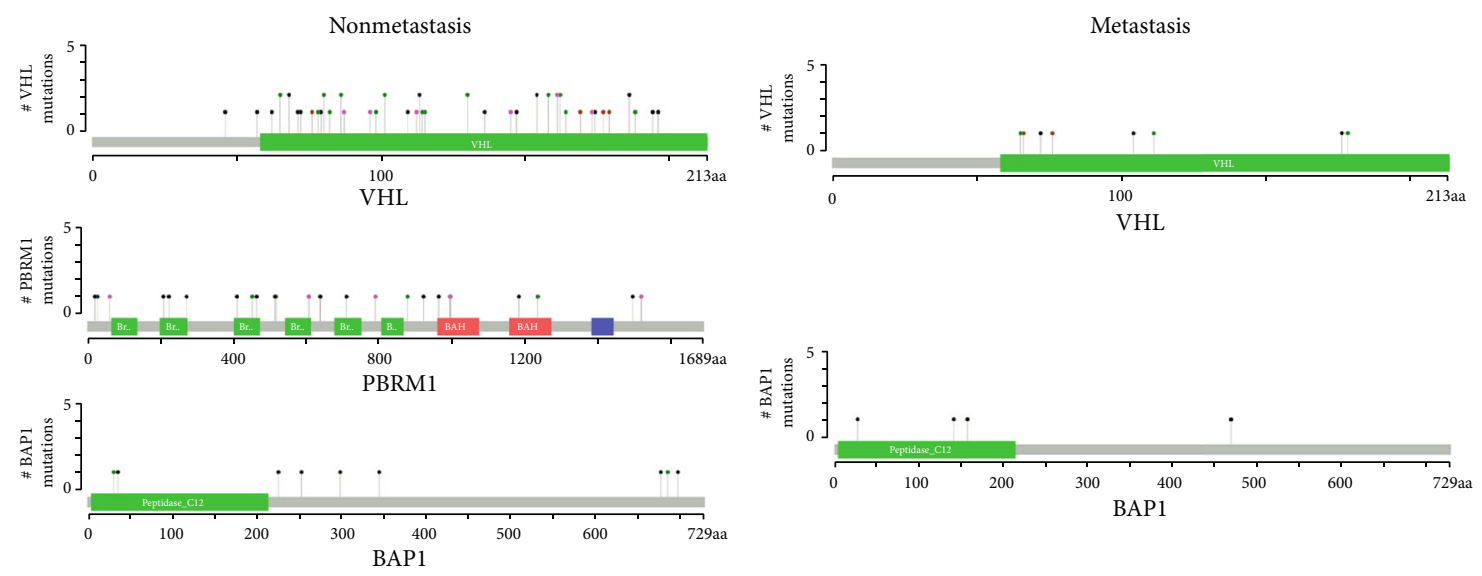

(c)

FIGURE 4: Analysis of differential mutation genes. (a) 101 mutated genes of different frequencies between the metastatic and nonmetastatic groups. (b) The location of identified TOP1 and SNCAIP variants. (c) The distribution of identified VHL, PBRM1, and BAP1 variants in the nonmetastatic (left) and metastatic (right) groups. The grey bar represents the entire protein marked with an amino acid number. The height of the grey line indicates the number of a specific mutation, and the colored circle on the grey line represents the corresponding mutation types. Green: missense; black: truncating; brown: inframe indels; purple: other.

TABLE 2: Genes with different mutated frequencies in ccRCC with or without metastasis.

\begin{tabular}{lccccc}
\hline & \multicolumn{2}{c}{$\begin{array}{c}\text { Metastasis } \\
(n=17)\end{array}$} & \multicolumn{2}{c}{$\begin{array}{c}\text { Nonmetastasis } \\
(n=89)\end{array}$} & $\begin{array}{c}P \\
\text { value }\end{array}$ \\
Gene & $\begin{array}{c}\text { Samples } \\
\text { with } \\
\text { mutation }\end{array}$ & $\begin{array}{c}\text { Samples } \\
\text { without } \\
\text { mutation }\end{array}$ & $\begin{array}{c}\text { Samples } \\
\text { with }\end{array}$ & $\begin{array}{c}\text { Samples } \\
\text { mutation }\end{array}$ & $\begin{array}{c}\text { without } \\
\text { mutation }\end{array}$ \\
\hline TOP1 & 3 & 14 & 0 & 89 & 0.004 \\
SNCAIP & 3 & 14 & 0 & 89 & 0.004 \\
PBRM1 & 0 & 17 & 24 & 65 & 0.011 \\
PRDM1 & 2 & 15 & 0 & 89 & 0.024 \\
CDKN1A & 2 & 15 & 0 & 89 & 0.024 \\
SMAD4 & 2 & 15 & 1 & 88 & 0.066 \\
PTK2 & 2 & 15 & 1 & 88 & 0.066 \\
LATS2 & 2 & 15 & 1 & 88 & 0.066 \\
KDM6A & 2 & 15 & 1 & 88 & 0.066 \\
TCF3 & 2 & 15 & 2 & 87 & 0.120 \\
WRN & 1 & 16 & 0 & 89 & 0.160 \\
TGFBR1 & 1 & 16 & 0 & 89 & 0.160 \\
TEK & 1 & 16 & 0 & 89 & 0.160 \\
TCF7L2 & 1 & 16 & 0 & 89 & 0.160 \\
SPOP & 1 & 16 & 0 & 89 & 0.160 \\
\hline & & & & &
\end{tabular}

proliferation by trapping Top1 on the DNA to stop the DNA replication process. However, the TOP1 alterations we identified (including p.His399Arg, p.Thr154Lys, and p.Gln713Glu) were not function clarified yet, so the potential response to the camptothecin-related drugs could not be predicted. The other unique marker of metastasis we identified was SNCAIP, a gene encoded synuclein alpha interacting protein, which was mainly related to Parkinson disease. Due to the deficiency of research, the oncogenic or tumor suppressor role of SNCAIP was not well depicted [16]. Promoter hypermethylation of SNCAIP could serve as a marker for colorectal cancer identification [17]. A previous study revealed that SNCAIP mutations were uniquely found in the diabetic group in pancreatic cancer, mainly involved in immune-related pathways [18]. In bladder cancer, SNCAIP was dysregulated and identified as a hub gene for predicting disease progression and prognosis [19]. Meanwhile, SNCAIP was rarely mutated $(0.44 \%)$ but highly amplified $(11.61 \%)$ in ccRCC in the TCGA database. So, it is worthy of additional studies on SNCAIP function and potentially drug development in ccRCC. We also identified CDKN1A alterations in the metastatic groups, and loss of CDKN1A had been proved to be an unfavorable predictor of prognosis in chromophobe renal cell carcinoma patients [20].

The application of immune checkpoint inhibitors (ICIs) in ccRCC has been rapidly progressing in the past years, so 


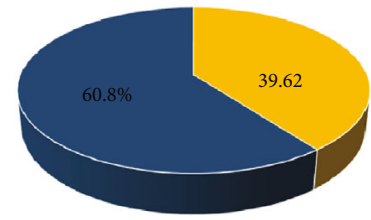

DNA damage repair gene -
DNA damage repair gene +

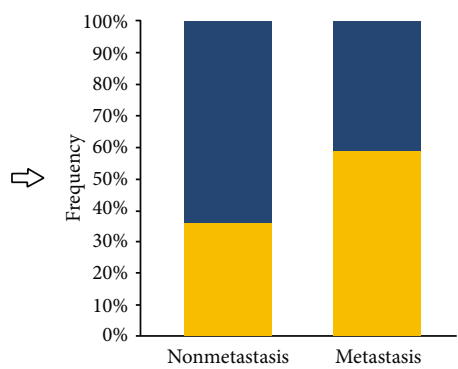

(a)

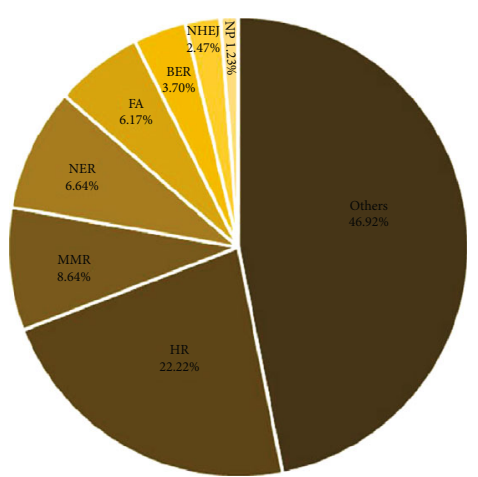

(b)



(c)

FIgURE 5: Analysis of somatic alterations in the DDR pathway. (a) The frequency of DDR mutations across ccRCC patients with or without metastasis. (b) The frequency of specific mutated pathway in DDR. (c) Frequency of mutated DDR genes with known deleterious variants. HR: homology recombination; MMR: mismatch repair; NER: nucleotide excision repair; FA: Fanconi anemia; BER: base excision repair; NHEJ: nonhomology end joining; NP: nucleotide pool maintenance.

TABLE 3: Details of pathogenic or likely pathogenic variant carriers.

\begin{tabular}{|c|c|c|c|c|c|c|}
\hline $\mathrm{ID}^{\#}$ & Age & Family history & Gene & Exon & Nucleotide change & Amino acid change \\
\hline 005 & 50 & No & $R A D 51 D$ & 4 & c.271_272insTA & p.Lys91fs \\
\hline 016 & 45 & No & MUTYH & 2 & c. $55 \mathrm{C}>\mathrm{T}$ & p.Arg19Ter \\
\hline 022 & 34 & Grandmother & $V H L$ & 2 & c. $345 \mathrm{C}>\mathrm{G}$ & p.His115Gln \\
\hline 024 & 54 & Father & $N B N$ & 5 & c.499_500insT & p.Cys167fs \\
\hline 037 & 37 & No & MUTYH & 13 & c. $1214 \mathrm{C}>\mathrm{T}$ & p.Pro405Leu \\
\hline 041 & 66 & No & $B R C A 2$ & 11 & c.3098_3099delAT & p.Asp1033fs \\
\hline 056 & 67 & No & $N B N$ & 14 & c.2167delC & p.Leu723Ter \\
\hline $058^{*}$ & 38 & No & ATM & 10 & c.1402_1403delAA & p.Lys468fs \\
\hline 069 & 65 & Brother & RAD50 & 12 & c.1821_1822insA & p.Lys608fs \\
\hline $076^{*}$ & 47 & No & ATM & 10 & c.1402_1403delAA & p.Lys468fs \\
\hline 092 & 55 & No & $\mathrm{FH}$ & 3 & c. $301 \mathrm{C}>\mathrm{T}$ & p.Arg101Ter \\
\hline
\end{tabular}


the potential response biomarkers are arresting significant interests. The primary known molecular biomarkers for ICIs were MSI-H and TMB, respectively. Previous research had found that MSI-H was rare $(0.6 \%)$ in RCC, which was also proved in our cohort as no MSI-H tumor was identified in the 106 ccRCC [21]. Research based on unmatched primary and metastatic non-small-cell lung carcinoma found a higher TMB trend in metastatic tumors [22]. However, no significant difference was found between metastatic and nonmetastatic tumors in our study. Limited by the sample sizes, we suggested that further research would be needed to reveal the difference in TMB between metastatic and nonmetastatic ccRCC. Furthermore, recent research found a correlation between DDR gene alterations and ICIs [23]. A retrospective analysis found that $5 \%$ and $16 \%$ ccRCC patients had germline and tumor variants in DDR genes, respectively. Meanwhile, oncogenic DDR gene variants were associated with better overall survival in immune-oncology but not in the tyrosine kinase inhibitor cohort [24]. A relatively higher incidence of DDR mutation was identified in the metastatic groups $(58.82 \%$ versus $35.96 \%)$ in our research. Whether metastatic ccRCC patients with DDR mutation would benefit from ICIs, PARP inhibitor, or platinum needed further investigation.

Hereditary kidney cancer was estimated to account for $5 \%-8 \%$ of newly diagnosed kidney cancer. A recently published study found that $9.5 \%$ of Chinese sporadic earlyonset (below 46 years old at diagnosis) had P/LP variants in 10 genes and a significant correlation to family cancer history in second-degree relatives [25]. In our cohort, $10.38 \%$ of sporadic ccRCC patients had P/LP germline variants, and the main reason for the higher frequency may be because of the more widely analyzed genes involved. Notably, $81.82 \%$ (9/11) P/LP variants were in genes related to the DDR repair pathway. Of patients with P/LP variants, 7 (63.64\%) would not have been referred for genetic evaluation according to NCCN and Chinese kidney cancer guidelines.

\section{Conclusions}

This present study demonstrated the genetic landscape of Chinese nonmetastatic and metastatic ccRCC patients. Different prevalence of genes, especially TOP1 and SNCAIP, was suggested to associate with metastasis. On the contrary, PBRM1 was correlated with nonmetastatic disease. A higher incidence of DNA damage repair gene alteration was identified in the metastatic disease. Further development of target drugs on TOP1, SNCAIP, and DDR might be useful for Chinese metastatic ccRCC patients.

\section{Data Availability}

The data used to support the findings of this study are available from the corresponding author upon request.

\section{Conflicts of Interest}

The authors declared that they have no conflicts of interest in this work.

\section{Authors' Contributions}

All authors contributed to this study design. Hui Meng conducted the literature search. Xuewen Jiang conducted the histological evaluation and immunohistochemical evaluation. Jianfeng Cui prepared patient samples. Benkang Shi performed the sequencing analysis. Qi Liu analyzed the data. All authors discussed the results and approved the final manuscript.

\section{Supplementary Materials}

Clinical information of metastasis (Supplementary Table S1), targeted sequencing genes in the article (Supplementary Table S2), differential mutations between our data and TCGA dataset (Supplementary Table S3), variant allele fraction of the mutations (Supplementary Table S4), and driver genes (Supplementary Table S5) are provided in supplementary tables. (Supplementary materials)

\section{References}

[1] W. Chen, R. Zheng, P. D. Baade et al., "Cancer statistics in China, 2015," CA: a Cancer Journal for Clinicians, vol. 66, no. 2, pp. 115-132, 2016.

[2] M. I. Carlo, N. Khan, A. Zehir et al., "Comprehensive genomic analysis of metastatic non-clear-cell renal cell carcinoma to identify therapeutic targets," JCO Precision Oncology, vol. 3, no. 3, pp. 1-18, 2019.

[3] M. Meskawi, M. Sun, Q. D. Trinh et al., "A review of integrated staging systems for renal cell carcinoma," European Urology, vol. 62, no. 2, pp. 303-314, 2012.

[4] Cancer Genome Atlas Research Network, W. M. Linehan, P. T. Spellman et al., "Comprehensive molecular characterization of clear cell renal cell carcinoma," Nature, vol. 499, no. 7456, 2013.

[5] N. Dizman, E. J. Philip, and S. K. Pal, "Genomic Profiling in Renal Cell Carcinoma," Nature Reviews Nephrology, vol. 16, no. 8, pp. 435-451, 2020.

[6] G. Guo, Y. Gui, S. Gao et al., "Frequent mutations of genes encoding ubiquitin-mediated proteolysis pathway components in clear cell renal cell carcinoma," Nature Genetics, vol. 44, no. 1, pp. 17-19, 2011.

[7] G. de Velasco, S. A. Wankowicz, R. Madison et al., "Targeted genomic landscape of metastases compared to primary tumours in clear cell metastatic renal cell carcinoma," British Journal of Cancer, vol. 118, no. 9, pp. 1238-1242, 2018.

[8] S. Richards, on behalf of the ACMG Laboratory Quality Assurance Committee, N. Aziz et al., "Standards and guidelines for the interpretation of sequence variants: a joint consensus recommendation of the American College of Medical Genetics and Genomics and the Association for Molecular Pathology," Genetics in Medicine, vol. 17, no. 5, pp. 405-423, 2015.

[9] M. M. Li, M. Datto, E. J. Duncavage et al., "Standards and guidelines for the interpretation and reporting of sequence variants in cancer: a joint consensus recommendation of the Association for Molecular Pathology, American Society of Clinical Oncology, and College of American Pathologists," The Journal of Molecular Diagnostics, vol. 19, no. 1, pp. 4-23, 2017. 
[10] Z. R. Chalmers, C. F. Connelly, D. Fabrizio et al., "Analysis of 100,000 human cancer genomes reveals the landscape of tumor mutational burden," Genome Medicine, vol. 9, no. 1, p. 34, 2017.

[11] B. Liang, J. Zhao, and X. Wang, "Clinical performance of E2Fs 1-3 in kidney clear cell renal cancer, evidence from bioinformatics analysis," Genes \& Cancer, vol. 8, no. 5-6, pp. 600607, 2017.

[12] P. Kapur, S. Peña-Llopis, A. Christie et al., "Effects on survival of BAP1 and PBRM1 mutations in sporadic clear-cell renalcell carcinoma: a retrospective analysis with independent validation," The Lancet Oncology, vol. 14, no. 2, pp. 159-167, 2013.

[13] D. Miao, C. A. Margolis, W. Gao et al., "Genomic correlates of response to immune checkpoint therapies in clear cell renal cell carcinoma," Science, vol. 359, no. 6377, pp. 801-806, 2018.

[14] X. D. Liu, W. Kong, C. B. Peterson et al., "PBRM1 loss defines a nonimmunogenic tumor phenotype associated with checkpoint inhibitor resistance in renal carcinoma," Nature Communications, vol. 11, no. 1, p. 2135, 2020.

[15] D. Gupta, I. B. Bronstein, and J. A. Holden, "Expression of DNA topoisomerase I in neoplasms of the kidney: correlation with histological grade, proliferation, and patient survival," Human Pathology, vol. 31, no. 2, pp. 214-219, 2000.

[16] Y. X. Li, Z. W. Yu, T. Jiang et al., "SNCA, a novel biomarker for group 4 medulloblastomas, can inhibit tumor invasion and induce apoptosis," Cancer Science, vol. 109, no. 4, pp. 12631275, 2018.

[17] G. E. Lind, S. A. Danielsen, T. Ahlquist et al., "Identification of an epigenetic biomarker panel with high sensitivity and specificity for colorectal cancer and adenomas," Molecular Cancer, vol. 10, no. 1, p. 85, 2011.

[18] K. Wang, W. Zhou, P. Meng et al., "Immune-related somatic mutation genes are enriched in PDACs with diabetes," Translational Oncology, vol. 12, no. 9, pp. 1147-1154, 2019.

[19] H. Yin, C. Zhang, X. Gou, W. He, and D. Gan, "Identification of a 13-mRNA signature for predicting disease progression and prognosis in patients with bladder cancer," Oncology Reports, vol. 43, no. 2, pp. 379-394, 2020.

[20] R. Ohashi, S. Angori, A. A. Batavia et al., "Loss of CDKN1A mRNA and protein expression are independent predictors of poor outcome in chromophobe renal cell carcinoma patients," Cancers (Basel), vol. 12, no. 2, p. 465, 2020.

[21] A. Vanderwalde, D. Spetzler, N. Xiao, Z. Gatalica, and J. Marshall, "Microsatellite instability status determined by next-generation sequencing and compared with PD-L1 and tumor mutational burden in 11,348 patients," Cancer Medicine, vol. 7, no. 3, pp. 746-756, 2018.

[22] M. K. Stein, M. Pandey, J. Xiu et al., “Tumor mutational burden is site specific in non-small-cell lung cancer and is highest in lung adenocarcinoma brain metastases," JCO Precision Oncology, vol. 3, no. 3, pp. 1-13, 2019.

[23] M. Y. Teo, K. Seier, I. Ostrovnaya et al., "Alterations in DNA damage response and repair genes as potential marker of clinical benefit from PD-1/PD-L1 blockade in advanced urothelial cancers," Journal of Clinical Oncology, vol. 36, no. 17, pp. 1685-1694, 2018.
[24] Y. Ged, J. Chaim, A. Knezevic et al., "Alterations in DNA damage repair (DDR) genes and outcomes to systemic therapy in 225 immune-oncology (IO) versus tyrosine kinase inhibitor (TKI) treated metastatic clear cell renal cell carcinoma (mccRCC) patients (pts)," Journal of Clinical Oncology, vol. 37, 7_suppl, p. 551, 2019.

[25] J. Wu, H. Wang, C. J. Ricketts et al., "Germline mutations of renal cancer predisposition genes and clinical relevance in Chinese patients with sporadic, early-onset disease," Cancer, vol. 125, no. 7, pp. 1060-1069, 2019. 